Pacific Journal of Mathematic 


\title{
HOMOMORPHISMS OF NEAR-RINGS OF CONTINUOUS FUNCTIONS
}

\author{
LI PI SU
}

\begin{abstract}
In recent papers Chew has found a class of topological rings such that if $E$ is one of them, then a space is $E$-compact if and only if every $E$-homomorphism on $C(X, E)$ has a onepoint support. We generalize this result to a class of topological near-rings. We also have found some topological near-rings which belong to this class.
\end{abstract}

Chew [5] proved that for the class of $\alpha$-topological rings, $\mathscr{E}, X$ is $E$-compact, $E \in \mathscr{E}$, if and only if every $E$-homomorphism on $C(X, E)$ has a one-point support. He also gave a "determination theorems."

The purpose of this paper is to show that the above results hold true for a class of topological near-rings. Since our arguments are almost identical with those of [5], we shall give only the statement of the results and the necessary definitions with a very brief indication of some proofs.

\section{Preliminaries.}

Definition 1.1. A near-ring is a triple $\{R,+, \cdot\}$ where $R$ is a nonempty set, each of + and $\cdot$ is an associative binary operation on $R$ such that $\{R,+\}$ is a group (need not be abelian) with identity 0 , and the following are satisfied,

(a) for each $x, y$, and $z \in R, x \cdot(y+z)=x \cdot y+x \cdot z$, and

(b) for each $x \in R, 0 \cdot x=0$. See [1].

Note that in [2] this type of near-ring is called $D$-ring. Examples can be found in [2].

DEFINITION 1.2. A near-ring $R$ that contains more than one element is said to be a division near-ring, or near-field if the set $R^{\prime}$ of nonzero elements is a multiplicative group; and 1 denotes the unity of $R^{\prime}$. See [8] and [9].

DEFINITION 1.3. A topological near-ring is a quadruple $\{R,+, \cdot, \mathscr{T}\}$ such that $\{R,+, \cdot\}$ is a near-ring, and $\mathscr{T}$ is a Hausdorff topology on $R$ such that the mappings

$$
f: R \times R \rightarrow R \text { defined by } f((x, y))=x+y
$$

and

$$
g: R \times R \rightarrow R \text { defined by } g((x, y))=x \cdot y
$$


are continuous. Compare [1]; and a topological near-field is a topological near-ring $\{R,+, \cdot, \mathscr{T}\}$ such that the mapping

$$
h:\left(R^{\prime}, R^{\prime} \mid \mathscr{T}\right) \rightarrow\left(R^{\prime}, R^{\prime} \mid \mathscr{T}\right) \text { defined by } h(x)=x^{-1}
$$

is continuous, where $x^{-1}$ in $R^{\prime}$ is the inverse of $x$ under.. See [13, p. 283].

DEFINITION 1.4. A near-ring homomorphism is a mapping $\phi$ of a near-ring $R$ into a near-ring $R_{0}$ such that

$$
\begin{aligned}
& \phi\left(\gamma_{1}+\gamma_{2}\right)=\phi\left(\gamma_{1}\right)+\phi\left(\gamma_{2}\right) \\
& \phi\left(\gamma_{1} \cdot \gamma_{2}\right)=\phi\left(\gamma_{1}\right) \cdot \phi\left(\gamma_{2}\right)
\end{aligned}
$$

for all $\gamma_{1}$ and $\gamma_{2}$ in $R$. See [3].

A subset $I$ of a near-ring $R$ is said to be a two-sided ideal, or simply an ideal if $(I,+)$ is a normal subgroup of $R$ such that

(1) $R I \subset I$

(2) $\left(\gamma_{1}+t\right) \gamma_{2}-\gamma_{1} \gamma_{2}$ is in $I$ if $\gamma_{1}$ and $\gamma_{2}$ are in $R$ and $t$ is in $I$. See [3].

Then we can easily show that the kernel of a homomorphism is an ideal. Note that $x \cdot 0=0$ for any $x$ in $R$ can be shown by using the left distributive law.

For notation and terminology, basic facts concerning $E$-compact and $E$-completely regular spaces, and structures of continuous functions we refer to [10], [11] and [5].

Let $C(X, E)$ be the set of all continuous functions from $X$ into the topological near-ring $E$, and the operations are defined pointwisely. Then $C(X, E)$ is a near-ring.

Let $H(X, E)$ be the space of all $E$-homomorphisms on $C(X, E)$ endowed with the relative product topology from $E^{(C(X, E)}$, and $\sigma$ be the parametric (evaluation) map corresponding to $C(X, E)$; i.e., $(\sigma(x))(f)=f(x)$ for each $x$ in $X$ and $f$ in $C(X, E)$. By an $E$-homomorphism we mean a homomorphism $\dot{\rho}$ from $C(X, E)$ into $E$ such that $\phi(e)=e$ for all $e$ in $E$ where $e$ is the constant function, $e[X]=\{e\}$.

We recall Theorems (2.1), (3.8) of [10].

Proposition 1.5. For any topological space $E$,

(a) A space $X$ is E-completely regular if and only if $\sigma$ is a homeomorphism.

(b) For any E-completely regular space $X, \beta_{E} X={ }_{e x t} c l_{P} \sigma[X]$, the closure of $\sigma[X]$ in $P=E^{(X, E)}$ 
(c) A space $X$ is E-compact if and only if $\sigma$ is a homeomorphism and $\sigma[X]$ is closed in $P$.

2. Representation theorems. In this section, $E$ is a topological near-ring.

Proposition 2.1. For any space $X$, the space $H(X, E)$ is closed in $E^{C(X, E)}$.

Proof. See [5, (2.1)].

The next proposition is to give a condition for topological nearrings such that $H(X, E)=c l_{P} \sigma[X]$.

Proposition 2.2. Suppose that $E$ is a topological near-ring with the property

$(\alpha)$ if $\phi$ in $H(X, E)$, then the family of zero-sets

$$
\{Z(f): f \in C(X, E), f \in \operatorname{ker} \dot{\phi}\}
$$

has the finite intersection property.

Then $\operatorname{cl}_{P} \sigma[X]=H(X, E)$ for any space $X$.

We shall call the topological near-ring with the property $(\alpha)$ an $\alpha$-topological near-ring.

THEOREM 2.3. Let $E$ be an $\alpha$-topological near-ring. An $E$ completely regular space $X$ is $E$-compact if and only if every $E$ homomorphism $\phi: C(X, E) \rightarrow E$ has a one-point support, $\left\{p_{0}\right\}$, in $X$.

More generally, for every $E$-completely regular space $X, E$ homomorphisms of $C(X, E)$ into $E$ correspond to the points of $\beta_{E} X$, the E-compactification of $X$.

Proof. Combining Prop. (1.5) and Prop. (2.2), we can easily prove the necessity; and use contrapositive to prove the sufficiency. As for the second part, we consider the natural correspondence between $C(X, E)$ and $C\left(\beta_{E} X, E\right)$. See [5, (2.3)].

In Theorem 2.3, we may give an additional condition on $E$, and then replace $E$-homomorphisms of $C(X, E)$ into $E$, by arbitrary homomorphisms of $C(X, E)$ into $E$. We have

CoRollaRY 2.4. Let $E$ be an $\alpha$-topological near-ring with the following property,

$(\beta)$ every nonzero endomorphism of $E$ is an automorphism. Then an E-completely regular space $X$ is E-compact if and only if 
every homomorphism $\dot{\text { from }} C(X, E)$ into $E$ has a one-point support.

Proof. sufficiency is clear.

Necessity. By assumption, each homomorphism $\phi$ from $C(X, E)$ into $E$ corresponds to an $E$-homomorphism $\zeta^{-1} \circ \phi$, where $\zeta$ is an automorphism of $E$ defined by $\zeta(e)=\phi(e)$ for each $e \in E$. The result follows immediately.

Now, we shall show the "determination theorems".

Corollary 2.5. For any $\alpha$-topological near-ring $E$, two $E$ compact spaces $X$ and $Y$ are homeomorphic if and only if the nearrings $C(X, E)$ and $C(Y, E)$ are E-isomorphic which means that there is an isomorphism $\phi$ from $C(X, E)$ onto $C(Y, E)$ with $\dot{\phi}(\boldsymbol{e})=\boldsymbol{e}$ for all $e$ in $E$.

Proof. The necessity is obvious, and the sufficiency is quite straightforward by combining Prop. (2.3) and the fact the $E$-isomorphism induces a one-to-one correspondence between $H(X, E)$ and $H(Y, E)$.

CoROLlary 2.6. Let $E$ be an $\alpha$-topological near-ring with property $(\beta)$. Then two E-compact spaces $X$ and $Y$ are homeomorphic if and only if the near-rings $C(X, E)$ and $C(Y, E)$ are isomorphic.

Proof. Use (2.4).

3. Remarks. In this section, we will see a sufficient condition for a topological near-ring to be an $\alpha$-topological near-ring, and some examples of $\alpha$-topological near-rings which satisfy the property $(\beta)$.

Proposition 3.1. Suppose that $E$ is a topological near-ring with the following properties:

(a) for any $\phi \in H(X, E), \phi(f)=0$ implies $Z(f) \neq \varnothing$.

(b) $E$ has a *function, i.e., there is a continuous function $x \rightarrow x^{*}$ of $E$ into itself such that $x x^{*}+y y^{*}=0$ implies $x=y=0$. Then $E$ is an $\alpha$-topological near-ring.

Proof is the same as that in $[5,(3.1)]$.

Besides the $\alpha$-topological rings which, of course, are $\alpha$-topological near-rings shown in [5, $\S 3]$, we have the following $\alpha$-topological near-rings.

An ordered near-field is defined in similar fashion as an ordered 
field, see [8, (2.1)]. A topological ordered near-field is an ordered near-field whose topology is defined in (1.3).

Proposition 3.2. Any topological ordered near-field, E, satisfies properties (a) and (b) in (3.1).

Proof. (a) Suppose $f \in C(X, E)$ and $Z(f)=\varnothing$. Then $f^{-1}$ defined by $f^{-1}(x)=[f(x)]^{-1}$ for each $x$ in $X$ is in $C(X, E)$, and $f \cdot f^{-1}=1$. Hence $f$ cannot be in any proper ideal of $C(X, E)$. If $\psi$ is a nonzero homomorphism from $C(X, E)$ into $E$, then ker

$$
\psi=\{h \in C(X, E): \psi(h)=0\}
$$

is a proper ideal of $C(X, E)$. Hence $f \notin$ ker $\psi$ which is a contradiction.

(b) Consider the identity mapping for ${ }^{*}$-function, i.e., $x^{*}=x$. Since $E$ is an ordered near-field $x x^{*}+y y^{*}=x^{2}+y^{2}=0$ implies $x=y=0$.

Proposition 3.3. Let $E$ be a near-field with discrete topology. Then $E$ is an $\alpha$-topological near-ring.

Proof. We shall prove this by induction. As the proof in (3.2) (a), if $f$ is in $C(X, E)$ with $Z(f)=\varnothing$, then $f$ does not belong to any kernel of element of $H(X, E)$. Thus, if $f_{1}$ in $C(X, E)$ with $\phi\left(f_{1}\right)=0$, then $Z\left(f_{1}\right) \neq \varnothing$. Assume that for $k=n-1, f_{1}, \cdots, f_{n-1} \in \operatorname{ker} \phi$, $\bigcap_{i=1}^{n-1} Z\left(f_{i}\right) \neq \varnothing$, but $f_{1}, \cdots, f_{n} \in$ ker $\phi$ with $\bigcap_{i=1}^{n} Z\left(f_{i}\right)=\varnothing$. Let $G_{k}=\bigcap_{i=1}^{k-1} Z\left(f_{i}\right) \backslash Z\left(f_{k}\right), k=2, \cdots, n$, and

$$
g_{k}(x)=\left\{\begin{array}{ll}
{\left[f_{k}(x)\right]^{-1}} & \text { if } x \in G_{k} \\
0 & \text { if } x \notin G_{k}
\end{array} .\right.
$$

Then since $G_{k}$ is both open and closed (as each $Z\left(f_{i}\right)$ is), $g_{k} \in C(X, E)$. Define $f=f_{1}+g_{2} f_{2}+\cdots+g_{n} f_{n}$. Then we can easily show that $Z(f)=\varnothing$. But that $\phi(f)=\phi\left(f_{1}\right)+\phi\left(g_{2}\right) \cdot \phi\left(f_{2}\right)+\cdots+\phi\left(g_{n}\right) \cdot \phi\left(f_{n}\right)=0$ implies $Z(f) \neq \varnothing$. This is a contradiction. Thus $\bigcap_{i=1}^{n} Z\left(f_{i}\right) \neq \varnothing$.

Finally, since the kernel of a homomorphism of near-ring is an ideal and in a near-field, there is no proper ideal hence each nonzero endomorphism of a near-field is an automorphism. Therefore by (3.2) and (3.3) a topological ordered near-field and a near-field with discrete topology have the properties $(\alpha)$ and $(\beta)$. 


\section{REFERENCES}

1. J. C. Beidleman and R. H. Cox, Topological near-rings, Sond. Arch. Math., 18 (1967), 485-492.

2. G. Berman and R. J. Silverman, Near-rings, Amer. Math. Mon., 66 (1959), 23-34. 3. D. W. Blackett, Simple and semisimple near-rings, Proc. Amer. Math. Soc., 4 (1953), 772-785.

4. Robert L. Blefko, Structures of continuous functions VII, Proc. Kon. Acad. Van. Vetench A, 71 (1968), 438-441.

5. Kim-Peu Chew, Structure of continuous functions IX homomorphisms of some functions rings, to appear in the Bull. of Poland Acad. of Sci.

6. L. Gillman and M. Jerison, Rings of Continuous Functions, D. Van Nostrand, (1960).

7. E. Hewitt, Rings of real-valued continuous functions I, Trans. Amer. Soc., 64 (1948), 54-99.

8. William Kerby, Angeordnete Fastkorper, Abh. Math. Sem. Univ. Hamburg. 32 (1968), 135-146.

9. Steve Ligh, On division near-rings, to appear.

10. S. Mrowka, Further results on E-compact spaces, Acta. Math., 120 (1968), 161-185.

11. - Structures of continuous functions $I$, to appear.

12. - Structures of continuous functions IV, Rings and lattices of integervalued continuous functions, Proc. Kon. Wetensch A 68 (1965), 74-82.

13. H. Wefelscheid, Vervollständigung topologischer Fastkörper, Math. Zeitschr 99, (1967), 279-298.

Received October 14, 1970.

The University of OKLAhoma 


\section{PACIFIC JOURNAL OF MATHEMATICS}

\section{EDITORS}

H. SAMELSON

Stanford University

Stanford, California 94305

C. R. Hовву

University of Washington

Seattle, Washington 98105
J. DUGUNDJI

Department of Mathematics

University of Southern California

Los Angeles, California 90007

RICHARD ARENS

University of California

Los Angeles, California 90024

\section{ASSOCIATE EDITORS}
E. F. BECKENBACH
B. H. NeumanN
F. WOLF
K. YoSHIDA

\section{SUPPORTING INSTITUTIONS}

UNIVERSITY OF BRITISH COLUMBIA

CALIFORNIA INSTITUTE OF TECHNOLOGY

UNIVERSITY OF CALIFORNIA

MONTANA STATE UNIVERSITY

UNIVERSITY OF NEVADA

NEW MEXICO STATE UNIVERSITY

OREGON STATE UNIVERSITY

UNIVERSITY OF OREGON

OSAKA UNIVERSITY

UNIVERSITY OF SOUTHERN CALIFORNIA
STANFORD UNIVERSITY

UNIVERSITY OF TOKYO

UNIVERSITY OF UTAH

WASHINGTON STATE UNIVERSITY

UNIVERSITY OF WASHINGTON

AMERICAN MATHEMATICAL SOCIETY CHEVRON RESEARCH CORPORATION NAVAL WEAPONS CENTER 


\section{Pacific Journal of Mathematics}

\section{Vol. 38, No. $1 \quad$ March, 1971}

Bruce Alan Barnes, Banach algebras which are ideals in a Banach algebra ..... 1

David W. Boyd, Inequalities for positive integral operators............... 9

Lawrence Gerald Brown, Note on the open mapping theorem .............. 25

Stephen Daniel Comer, Representations by algebras of sections over Boolean

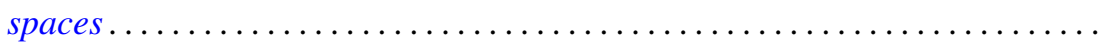

John R. Edwards and Stanley G. Wayment, On the nonequivalence of

conservative Hausdorff methods and Hausdorff moment sequences ........

P. D. T. A. Elliott, On the limiting distribution of additive functions $(\bmod 1) \ldots \ldots$

Mary Rodriguez Embry, Classifying special operators by means of subsets

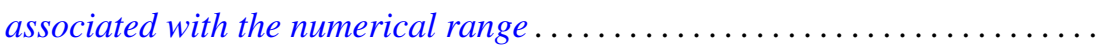

Darald Joe Hartfiel, Counterexamples to a conjecture of G. N. de Oliveira ......

C. Ward Henson, A family of countable homogeneous graphs...............

Satoru Igari and Shigehiko Kuratsubo, A sufficient condition for

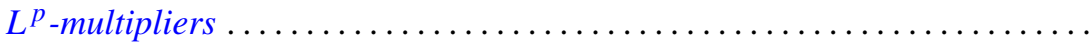

William A. Kirk, Fixed point theorems for nonlinear nonexpansive and

generalized contraction mappings............................

Erwin Kleinfeld, A generalization of commutative and associative rings ...... 95

D. B. Lahiri, Some restricted partition functions. Congruences modulo $11 \ldots \ldots 103$

T. Y. Lin, Homological algebra of stable homotopy ring $\pi *$ of spheres ....... 117

Morris Marden, A representation for the logarithmic derivative of a meromorphic function...........................

John Charles Nichols and James C. Smith, Examples concerning sum properties for metric-dependent dimension functions . .

Asit Baran Raha, On completely Hausdorff-completion of a completely

Hausdorff space.

M. Rajagopalan and Bertram Manuel Schreiber, Ergodic automorphisms and affine transformations of locally compact groups..........

N. V. Rao and Ashoke Kumar Roy, Linear isometries of some function

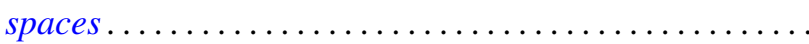

William Francis Reynolds, Blocks and F-class algebras of finite groups

Richard Rochberg, Which linear maps of the disk algebra are multiplicative ...

Gary Sampson, Sharp estimates of convolution transforms in terms of decreasing

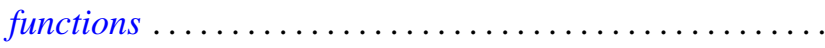

Stephen Scheinberg, Fatou's lemma in normed linear spaces

Ken Shaw, Whittaker constants for entire functions of several complex

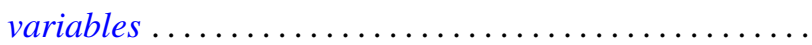

James DeWitt Stein, Two uniform boundedness theorems................ 251

$\mathrm{Li} \mathrm{Pi} \mathrm{Su,} \mathrm{Homomorphisms} \mathrm{of} \mathrm{near-rings} \mathrm{of} \mathrm{continuous} \mathrm{functions} \mathrm{.} \mathrm{.............} 261$

Stephen Willard, Functionally compact spaces, $C$-compact spaces and mappings of minimal Hausdorff spaces....................... 